\title{
Síndrome de Marfán
}

\author{
Pamela Oliva ${ }^{1}$, Regina Moreno A $^{2}$, M. Isabel Toledo $\mathbf{G}^{1}$, \\ Andrea Montecinos $\mathrm{O}^{\mathrm{a}}$, Juan Molina $\mathrm{P}^{1}$.
}

\section{Marfan Syndrome}

Marfan Syndrome is an autosomic dominant genetic disorder of the elastic fibers of connective tissue. Although neonatal and infant forms of the disease exist, the classic Marfan Syndrome is the most frequent form of presentation in childhood and adolescence, whith a hereditary background in 70 to $85 \%$ of cases. Due to the natural evolution of the disease, there is a progressive involvement of different organs or systems such as skeletal, cardiovascular, dura, ocular, skin-integument and lungs. However, the suspicion must arise on skeletal clinical aspects which are first evident signs. The cardiovascular involvement appears later but is the major life threatening complication. When suspecting Marfan phenotype, it is mandatory to apply Ghent criteria based on family history and clinical findings to establish the diagnosis. If diagnosis is confirmed, the severity of organ involvement must be assessed, to take preventive and/or therapeutic measures, including the search of new cases among relatives. When patients do not fulfill the diagnostic criteria, they must have a yearly evaluation considering the natural progressive evolution of the disease. The aim of this review is to spread and unify criteria on this disease whose diagnosis is eminently clinical, that requires early integral and updated management by a multidisciplinary group, to obtain the best quality of life and survival (Rev Méd Chile 2006; 134: 1455-64).

(Key w ords: Genetic diseases, inborn; heart defects, congenital; Marfan syndrome)

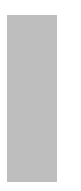

\begin{abstract}
Recibido el 16 de septiembre, 2005. Aceptado el 14 de junio, 2006.
${ }^{1}$ Unidad de Cardiología, Servicio de Pediatría, Hospital Dr. Hernán Henríquez A. Departamento de Pediatría y Cirugía Infantil, Facultad de Medicina, Universidad de la Frontera. ${ }^{2}$ Departamento de Ciencias Básicas, Facultad de Medicina, Universidad de la Frontera.

aAlumna Carrera de Enfermería, Universidad Mayor, Santiago.
\end{abstract}

$\mathrm{E}^{1}$ síndrome de Marfán es un trastorno genético autosómico dominante que afecta las fibras elásticas del tejido conectivo, manifestándose en aquellos sistemas u órganos que la contienen en mayor concentración, tales como el cardiovascular, esquelético, duramadre, ocular, piel, tegumentos y pulmón ${ }^{1,2}$.

La fibra elástica tiene como función la distensión y retracción, forma parte de la matriz extracelular de

Correspondencia a: Dra. Pamela Oliva N. Dinamarca 621, Ofic. 810. Temuco. Fono: (45) 204880. Fax: (45) 216210. E mail: moliva@ufro.cl los tejidos y está compuesta por elastina y una red de microfibrillas que sirve de armazón para el depósito de elastina y el ensamblaje de las fibras elásticas ${ }^{2-4}$. Esta red de microfibrillas está formada por fibrilina ${ }_{1}$, que está codificada por el gen $\mathrm{FBN}_{1}$ en el cromosoma 15q21, cuyo defecto se expresa mediante un efecto dominante negativo, es decir, en los heterocigotos, la fibrilina ${ }_{1}$ mutante destruye el ensamblaje de las microfibrillas normales, posiblemente, al actuar con los productos del alelo normal ${ }^{2}$. En este gen se han descrito más de 500 mutaciones y casi todas son únicas para un individuo o familia 
afectada ${ }^{5}$, dando origen a un defecto hereditario de la fibrilina, lo que ocasiona una formación de fibras elásticas anormales, con la consiguiente disfunción de los tejidos que la poseen. Además, se ha postulado que la fibrilina normal inhibińa el crecimiento de los huesos largos y que las fibras elásticas a través de su tensión controlańan el crecimiento de éstos, por lo tanto, al existir una alteración en estas estructuras se producińa el crecimiento óseo exagerado propio de la enfermedad ${ }^{6}$.

Incidencia y aspectos genéticos. La incidencia ha sido descrita entre 1/3.000 y 1/20.000 habitantes 2,5 . El 70\%-85\% de los casos de Marfán clásico son hereditarios. Se ha informado de casos en todas las razas y grupos étnicos ${ }^{2,7,8}$. La herencia es autosómica dominante con penetrancia casi completa, pero con expresión variable. La falla de la detección puede estar dada por la marcada variabilidad clínica (en la misma o diferentes familias), dependencia de la edad en la aparición de las manifestaciones clínicas (a mayor edad, mayor compromiso clínico) y la existencia de casos nuevos, producto de las mutaciones ${ }^{1,8}$.

Cuadro clínico. El diagnóstico se basa fundamentalmente en los hallazgos físicos. Existen 3 formas de presentación relacionadas estrechamente con la edad, con cuadros clínicos y pronósticos bien definidos: a) Marfán neonatal, b) Marfán infantil, c) Marfán clásico.

a. Marfán neonatal. Los escasos casos reportados han sido esporádicos. En la ecocardiografía prenatal se ha detectado cardiomegalia con insuficiencia tricuspídea severa. Al nacer, se evidencia alteraciones esqueléticas y de piel (extremidades largas con dedos finos, aspecto envejecido, piel laxa, hipotonía, alteraciones del tórax, contracturas en flexión, micrognatia) y cardiovasculares (insuficiencia mitral y tricuspídea severas, cardiomegalia, dilatación aórtica y pulmonar, arritmias, prolapso mitral y tricuspídeo, aneurismas masivos de la aorta ascendente y descendente). La muerte ocurre en horas o días por insuficiencia cardíaca ${ }^{9-12}$.

b. Marfán infantil. En el desarrollo de la enfermedad en este grupo, destaca la descripción hecha por Lipscomb en 40 niños menores de 16 años, con seguimiento de hasta 10 años a través de evalua- ción clínica y molecular ${ }^{1}$. En este estudio, la edad al diagnóstico de los casos esporádicos fue de $11,4 \pm 3,95$ años y en los familiares $7,3 \pm 5,23$ años. La aparición de las manifestaciones clínicas extracardíacas se describen en la Tabla 1, comprobándose el carácter evolutivo de ellas en relación a la edad. Las lesiones cardiovasculares se presentaron en $55 \%$ de los casos, todos asintomáticos, predominando la dilatación aórtica en $42 \%$ de los niños, con una edad media de detección de $11 \pm 3,9$ años. Se evidenció, además que al completarse la maduración y el fenotipo esquelético, la mayor parte de los pacientes tenían compromiso cardiovascular. Como manifestación asociada, describe retardo de la marcha y trastornos del aprendizaje. Otra revisión, de 86 casos, efectuada por Geva en 1990, que incluyó casos neonatales e infantiles ${ }^{13}$, se describe mayor frecuencia en hombres (2/3 de los casos), predominio de casos esporádicos (70\%) y edad media al diagnóstico de 3,2 meses; con compromiso esquelético en $100 \%$ de los pacientes y anomalías oculares en 83\%. Respecto del compromiso cardiovascular, las lesiones más frecuentes fueron: prolapso mitral, 96\%, dilatación raíz aórtica, 86\% e insuficiencia mitral, 84\%. La edad media de muerte fue de 16,3 meses. El 74\% presentó insuficiencia cardíaca y $21 \%$ requirió cirugía. En lactantes hubo mayor morbimortalidad por lesiones de la mitral y en la niñez-adolescencia por compromiso aórti$\mathrm{co}^{14}$. Estos datos documentan que el síndrome de Marfán neonatal-infantil tiene características clínicas y pronóstico claramente diferentes que los reportados en los pacientes de mayor edad.

\section{Tabla 1. Edad de aparición de las lesiones extracardíacas en niños}

(Lipscomb KJ et al, Arch DisChild 1997; 76: 41-46)

Alteraciones esqueléticas:

* Aracnodactilia: desde $\mathrm{RN}$

* Pie plano: desde 2-3 años

* Alteraciones faciales: desde los 3-4 años

* Paladar ojival: desde 3-4 años

* Escoliosis:

- Edad menor: 14 meses

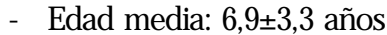

- Edad más frecuente: 10-14 años

* Deformidad tórax:

- Edad menor: 18 meses

- Edad máxima: pubertad 
c. Marfán clásico. Esta forma de presentación es la más frecuente y reconocida y se presenta en niños, adolescentes y adultos. Los criterios más recientes para el diagnóstico de la enfermedad son los proporcionados por la Nosología de Ghent en 1996 (Tabla 2), que se basan en la historia familiar-

Tabla 2. C riterios diagnósticos de síndrome de M arfán

(N osología de Ghent, 1996)

\begin{tabular}{|c|c|c|}
\hline Aspecto Evaluado & Criterio Mayor & Criterio Menor \\
\hline $\begin{array}{l}\text { Historia familiar/ } \\
\text { genética }\end{array}$ & \multicolumn{2}{|c|}{ 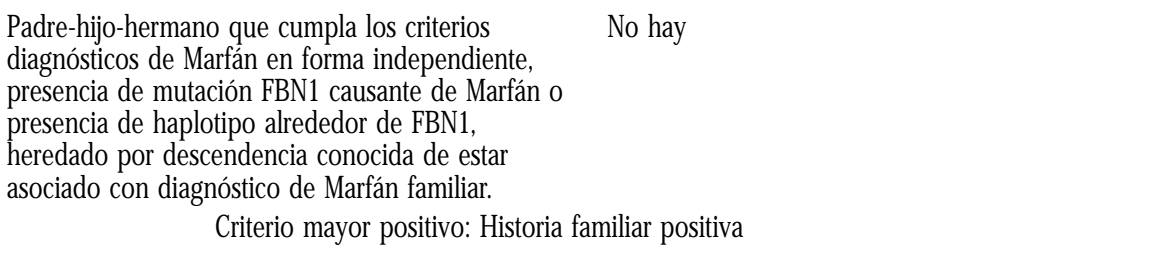 } \\
\hline Sistema esquelético & \multicolumn{2}{|c|}{ 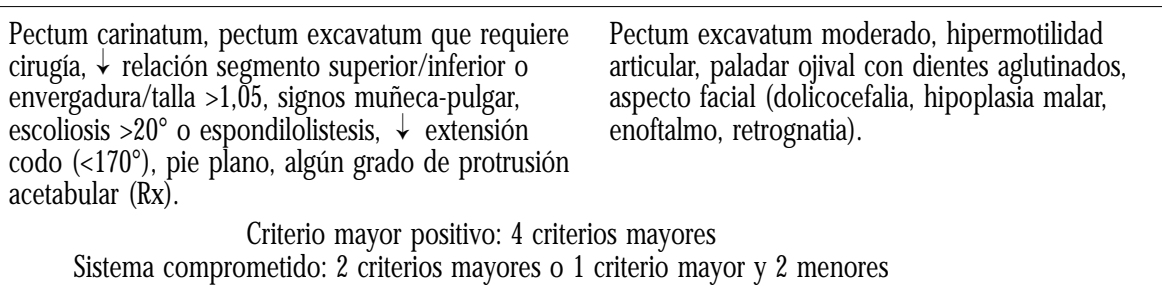 } \\
\hline Sistema cardiovascular & $\begin{array}{l}\text { Dilatación aorta ascendente con o sin insuficiencia } \\
\text { aórtica y al menos compromiso senos vasalva, } 0 \\
\text { disección aorta descendente }\end{array}$ & $\begin{array}{l}\text { Prolapso mitral con o sin insuficiencia, dilatación } \\
\text { arteria pulmonar, en ausencia de estenosis } \\
\text { pulmonar valvular o periférica u otra causa obvia, } \\
\text { en menor de } 40 \text { años, Calcificación de anillo mitral } \\
\text { en }<40 \text { años, dilatación/disección aorta } \\
\text { descendente } 0 \text { abdominal en }<50 \text { años. } \\
\text { io mayor } \\
\text { io menor }\end{array}$ \\
\hline Sistema pulmonar & No hay & $\begin{array}{l}\text { Neumotórax espontáneo, bulas apicales (RxTx). } \\
\text { hay } \\
\text { io menor }\end{array}$ \\
\hline Sistema ocular & $\begin{array}{l}\text { Subluxación de cristalino } \\
\qquad \text { Criterio mayor positivo: } 1 \text { crit } \\
\text { Sistema comprometido: } 2 \text { criter }\end{array}$ & $\begin{array}{l}\text { Comea plana, miopía axial, hipoplasia de iris } 0 \\
\text { músculo ciliar, hipoplasia causando miosis. } \\
\text { io mayor } \\
\text { s menores }\end{array}$ \\
\hline Piel & $\begin{array}{l}\text { Criterio mayor positivo: } 1 \\
\text { Sistema comprometido: } 1 \text { crit }\end{array}$ & $\begin{array}{l}\text { Estrias atróficas no asociadas a la baja de peso } 0 \\
\text { embarazo, hernia incisional o recurrente. } \\
\text { hay } \\
\text { io menor }\end{array}$ \\
\hline $\begin{array}{l}\text { Sistema nervioso } \\
\text { central }\end{array}$ & \multicolumn{2}{|c|}{$\begin{array}{l}\text { Criterio mayor positivo: } 1 \text { criterio mayor } \\
\text { Sistema comprometido: no hay }\end{array}$} \\
\hline \multicolumn{3}{|c|}{ Requisitos para el diagnóstico de Marfán } \\
\hline $\begin{array}{l}\text { 1. Caso índice: } \\
\text { Si historia genética } \\
2 \text { órganos/sistemas } \\
\text { de } 3^{\text {er órg./ sist. }} \\
\text { Si historia genética } \\
\text { Criterio mayor en } \\
\text { de } 2^{0} \text { órg./ sist. }\end{array}$ & $\begin{array}{l}\text { miliar es negativa: } \\
\text { on criterio mayor + compromiso } \\
\text { miliar es positiva: }\end{array}$ & $\begin{array}{l}\text { miliares de un caso índice: } \\
\text { ia de un criterio mayor en historia familiar }+1 \text { criterio } \\
\text { en órgano/sistema + compromiso de } 2^{\underline{0}} \\
\text { sistema }\end{array}$ \\
\hline
\end{tabular}



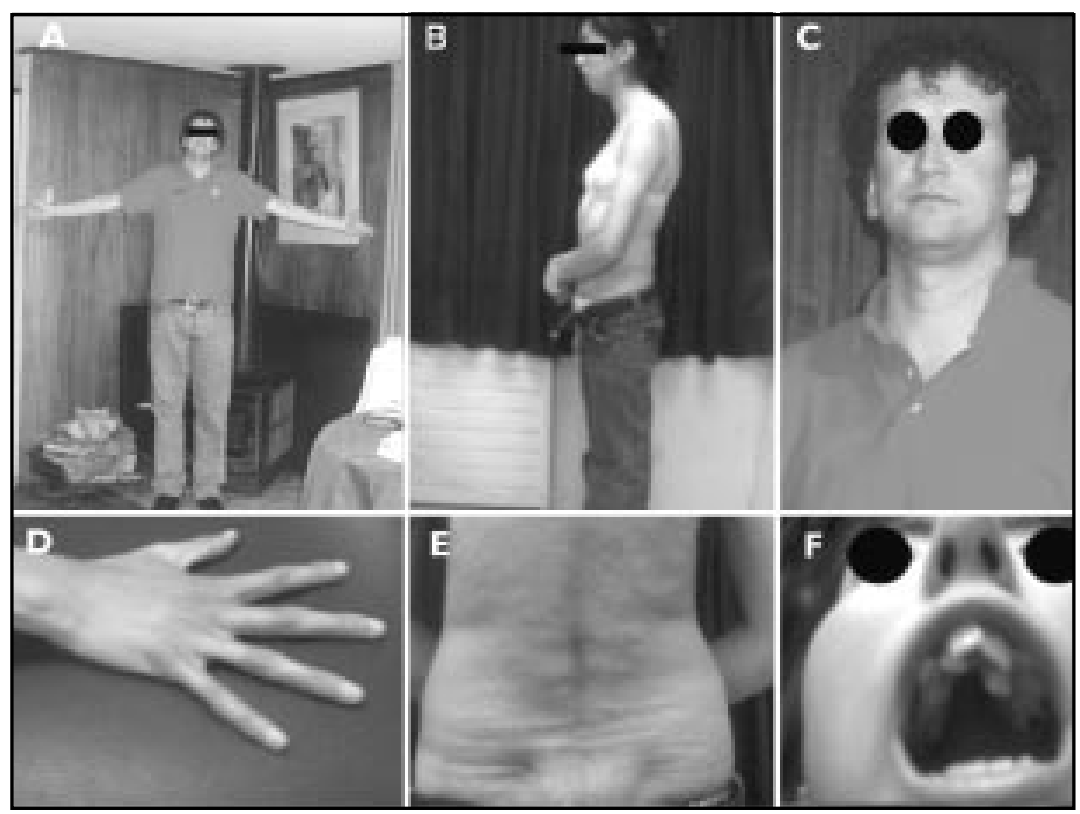

Figura 1. Manifestaciones clínicas síndrome Marfán.

A: Talla alta con mayor envergadura (nótese la cercanía de la cabeza del paciente al techo de la pieza). B: Xifosis. C: Fascia típica-dolicocefalia. D: Aracnodactilia. E: Estrías atróficas. F: Paladar ojival y dentadura aglutinada

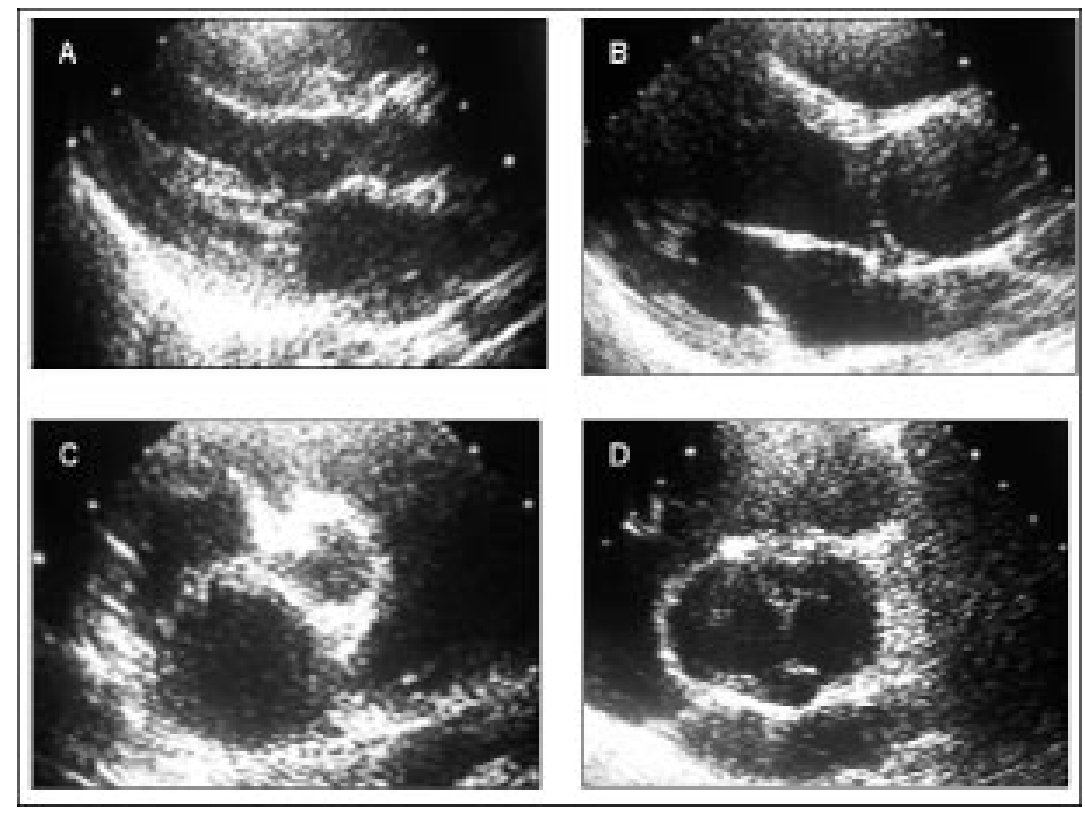

Figura 2. Imágenes ecocardiográficas de aorta en paciente normal y con síndrome de Marfán.

A: Eje largo paraesternal de aorta normal. B: Eje largo paraesternal de dilatación aórtica con prolapso, en paciente con síndrome de Marfán. C: Eje corto paraesternal de aorta normal. D: Eje corto paraesternal de dilatación aórtica en paciente con síndrome de Marfán. 
genética y el compromiso de los diferentes órganos o sistemas afectados ${ }^{15}$. Para evaluar los criterios de Ghent se debe considerar dos conceptos: uno es el criterio mayor, como evidencia de signo de alta especificidad de la enfermedad porque es infrecuente en otras condiciones o en la población general y otro es el compromiso de órgano o sistema, que indica afección pero con menor trascendencia en el diagnóstico. Las alteraciones esqueléticas son las más frecuentes y precoces de detectar y por ello son las que primero establecen la sospecha de la enfermedad. Son progresivas con la edad y se completan en la adolescencia. Destacan por su frecuencia la talla alta con mayor envergadura, pectum excavatum o carinatum, aracnodactilia, escoliosis, hipermotilidad articular y paladar ojival. La evaluación clínica se puede complementar con estudios radiográficos, principalmente con radiografía de pelvis, cuando se requiere detectar protrusión acetabular. Las lesiones cardiovasculares determinan el pronóstico del síndrome de Marfán, ya que son las que ocasionan la mayor mortalidad, con $70 \%$ $95 \%$ de los $\operatorname{casos}^{13}$, siendo más específica y frecuente la dilatación aórtica. Su incidencia depende de la edad: $40 \%-80 \%$ en niños y $80 \%-100 \%$ en adultos. La dilatación inicial compromete los senos de Valsalva y aorta ascendente y por ser progresiva se produce dilatación de la raíz aórtica, que lleva a insuficiencia valvular. Es de mal pronóstico por su carácter evolutivo, con riesgo de complicaciones como disección y ruptura. La dilatación se acelera con el estrés físico o emocional y con el embarazo. El compromiso de la válvula mitral (prolapso, insuficiencia) es frecuente pero menos grave. Las lesiones cardiovasculares son diagnosticadas rutinariamente por ecocardiografía transtorácica o transesofágica, esta última más resolutiva en estudios de arco aórtico ${ }^{16}$. La tomografía axial computada (TAC) es necesaria en pacientes que presenten dilatación aórtica acentuada (cercana a $50 \mathrm{~mm}$ por ecocardiografía en pacientes con talla adulta), para tener una medición más precisa, a objeto de determinar requerimientos de cirugía. La resonancia magnética (RM) se ha empleado en forma precoz para evaluar la distensibilidad aórtica, que es un indicador del estado de sus propiedades elásticas y por ello permite detectar compromiso antes que ocurra la dilatación. También se ha señalado como útil para detectar asimetría de la raíz aórtica, como signo temprano de riesgo de disección y por ser más precisa que la ecocardiografía en las lesiones de arco aórtico en adultos ${ }^{17-21}$. El compromiso ocular es frecuente (70\%) y progresivo. La lesión más específica para el diagnóstico es la subluxación del cristalino, sin embargo, es necesario también identificar vicios de refracción para preservar la máxima función visual. Existe mayor riesgo de glaucoma, cataratas y desprendimiento de retina, aun en ausencia de subluxación de cristalino. El estudio oftalmológico se realiza por evaluación del segmento anterior mediante biomicroscopia, córnea plana por queratometría y miopía axial por ultrasonido o refractometría. El daño pulmonar debe sospecharse con síntomas sugerentes de neumotórax 0 con radiografía de tórax que presente bulas apicales. La evaluación de piel y tegumentos en búsqueda de estrías atróficas no relacionadas a cambios bruscos de peso ni embarazo y hernias insicionales 0 recurrentes, debe ser rutinaria, ya que contribuye a aportar información para el caso de pacientes con cuadros clínicos sospechosos pero incompletos. La ectasia dural es una dilatación del saco dural principalmente lumbosacro, asintomática. En casos extremos puede provocar erosión vertebral y meningocele anterior. Se han descrito cefaleas posturales por extravasación de líquido cefalorraquídeo (LCR) e hipotensión intracraneal. El diagnóstico se puede realizar por TAC y $\mathrm{RM}^{22-24}$. Rose comparó las nosologías de Beighton 1988 (Berín) y de Paepe (Ghent) 1996 como criterios diagnósticos para Marfán, detectando que 19\% de los pacientes del criterio Berlín no cumplía los estándares de Ghent; además, mediante estudios dirigidos constató que la ectasia dural es la segunda manifestación más común, determinando 23\% del diagnóstico de Marfán bajo los criterios de Paepe, por lo que se recomendaría realizar su búsqueda en forma rutinaria ${ }^{25}$.

Diagnóstico diferencial. El diagnóstico diferencial se debe realizar con otras enfermedades genéticas que comprometen el tejido conectivo y enfermedades metabólicas que presentan algunos hallazgos clínicos semejantes pero presentados en forma aislada (Tabla 3). En nuestro medio, por no contar con estudios moleculares, tiene importancia la realización del test de nitroprusiato para descartar homocistinuria ${ }^{7}$. 


\section{Tabla 3. D iagnóstico diferencial}

1) Enfermedades por defecto FBN1 - cromosoma 15.

* Subluxación familiar cristalino.

* Dilatación/disección aórtica familiar aislada.

* Fenotipo MASS (prolapso mitral, dilatación aórtica, anomalías esqueléticas, piel laxa).

* Craneosinostosis marfanoide.

3) Enfermedades metabólicas.

* Homocistinuria (luxación cristalino, talla alta, alteraciones CV).
2) Enfermedad por defecto FBN2 - cromosoma 15.

* Aracnodactilia contractural congénita.

4) Otras.

* Hábito marfanoide familiar

* Enfermedad congénita polivalvular.

* Síndrome prolapso mitral familiar.

* Síndrome Stickler (ocular, esquelético, prolapso mitral).
Tratamiento y seguimiento. Se deben considerar numerosos y variados aspectos:

A. Prevención primaria: se debe plantear la realización de consejo genético considerando la tasa de recurrencias, opciones de reproducción y existencia de nuevos casos en otros familiares recordando la variabilidad de fenotipo dentro de la misma y entre familias ${ }^{5,7,26}$. Cada hijo de un individuo afectado presenta $50 \%$ de riesgo de heredar el síndrome de Marfán. El diagnóstico molecular prenatal no se encuentra disponible en forma rutinaria y rara vez se ha hecho diagnóstico por ecografía de fetos afectados.

\section{B. Prevención de complicaciones:}

1) Se requiere evaluación anual ortopédica, cardiovascular y oftalmológica para detectar la aparición de lesiones, la evolución de ellas cuando están presentes y para tratarlas oportunamente cuando lo requieren. Evaluación por genetista para confirmación y seguimiento. Realizar vigilancia pediátrica de los parámetros de crecimiento y desarrollo. Pautas de seguimiento profesional y guías anticipatorias por edad, principalmente destinadas a la educación de los padres y pacientes son recomendadas por el Comité de Genética de la Academia Americana de Pediatría 26.

2) La evaluación cardiológica debe ser tanto clínica como ecocardiográfica y su frecuencia dependerá tanto de la existencia de lesiones como de su severidad. Recomendaciones precisas de actividad física de acuerdo al grado de compromiso cardíaco se encuentran avaladas con evidencia IA y IIA ${ }^{27}$. En niños, es necesario focalizarlos hacia el desarrollo de habilidades y deportes como golf, marcha, pesca.
3) Efectuar profilaxis de endocarditis infecciosa cuando existe compromiso valvular, después de reemplazo de raíz aórtica o si hay válvula protésica $^{5}$.

4) Uso de betabloqueadores destinados a evitar la aparición de la dilatación aórtica en todos los pacientes con Marfán, o detener su progresión cuando ya ha comenzado para reducir el riesgo de disección. Un ensayo clínico aleatorio de tratamiento con propanolol en 70 adolescentes y adultos jóvenes con Marfán comparado con pacientes que no recibieron fármaco, demostró una reducción de la tasa de dilatación aóntica y menos complicaciones en el grupo tratado ${ }^{28}$. Otro estudio posterior mostró que la terapia con metoprolol y atenolol reducía significativamente el promedio de presión arterial y rigidez aórtica en pacientes con Marfán versus controles ${ }^{29}$. Estos estudios proporcionan fuerte evidencia de que los betabloqueadores deberían ser considerados en todos los pacientes con Marfán, particularmente en los más jóvenes. Su uso se fundamenta en las propiedades de disminuir el inotropismo, la frecuencia cardíaca, la presión arterial y la rigidez aórtica ${ }^{5,7,28,29}$ y debe ser precoz y a dosis adecuadas. Se recomienda mantener frecuencias cardíacas en reposo menores de $70 \mathrm{x}^{\prime}$ en adultos $\mathrm{y} 80 \mathrm{x}^{\prime}$ en niños $\mathrm{y}$ postejercicio menores de $100 \mathrm{x}^{\prime}$ en adultos $\mathrm{y}$ $110 x^{\prime}$ en niños, las cuales deberían ser monitorizadas con Holter. Considerar que hay pacientes no respondedores a esta terapia y los efectos adversos, como agravamiento de asma o letargia que pueda provocar trastornos del aprendizaje y bradicardia sintomática. En caso de contraindicación o falta de respuesta a los betabloqueadores se ha usado inhibidores de la enzima convertido- 
ra de angiotensina o antagonistas del calcio ${ }^{30}$, sin embargo, es necesario disponer de mayor número de ensayos clínicos que demuestren su utilidad.

5) El embarazo puede agravar las lesiones cardiovasculares por la presencia de un estado hiperdinámico, existiendo mayor riesgo de ruptura aneurismática; el riesgo es menor si el compromiso cardíaco es mínimo y la raíz aórtica es menor de $40 \mathrm{~mm}$. Se recomienda evitarlo si existe dilatación mayor de $44 \mathrm{~mm}$ o realizar cirugía preventiva antes del embarazo; con diámetros entre 40 y $44 \mathrm{~mm}$ se debe evaluar de acuerdo a la velocidad de dilatación y a la historia familiar. 5 Si hay dilatación moderada, realizar ecocardiografía frecuente para observar si hay progresión rápida de la lesión. Controlar efecto de betabloqueadores en el recién nacido. En la gestación, se ha recomendado también el uso de labetalol ${ }^{5}$. La vía del parto aún es motivo de debate, planteándose parto vaginal o cesárea de acuerdo a las lesiones existentes y su grado. Se debe evitar el estrés del parto con anestesia epidural y hacer esfuerzos por acortar la segunda fase del trabajo de parto ${ }^{30}$. La anestesia raquídea o epidural debe administrarse sólo después de considerar la presencia de ectasia dural ${ }^{5}$. Se han reportado casos de cesárea de urgencia y postenior cardiocirugía en casos de riesgo inminente de disección o ruptura aórtica.

6) Realización de fisioterapia para mejorar el tono muscular y actividad física moderada no traumática (natación, bicicleta) de acuerdo al compromiso cardíaco.

7) Frenación de la talla a través de inducción de pubertad precoz.

8) Buscar impedimentos del aprendizaje: trastorno visual, retardo motor, baja autoestima, efecto de medicamentos.

9) Apoyo sicológico para el niño y su familia. Evaluar aspectos positivos del paciente y capacidad de los padres de tratarlo como un niño normal, como la familia se comporta con un niño en condición crónica. Buscar grupos y literatura de apoyo. En adolescencia, apoyar con educación respecto de estilo de vida, limitaciones físicas y ocupacionales, sexualidad, reproducción y riesgos genéticos ${ }^{26}$.

10) Educación en síntomas de pesquisa de complicaciones como neumotórax, disección aórtica.
11) En pacientes con hallazgos incompletos que no cumplen con todos los criterios para la enfermedad, se sugiere reevaluar en forma anual, ya que por el carácter progresivo de esta patología pueden aparecer posteriormente.

C. Tratamiento de las complicaciones cardiovasculares: es siempre de tipo quirúrgico. En insuficiencia mitral, situación que se da especialmente en niños, se puede optar por una reparación valvular que se determina de acuerdo a los criterios generales para este tipo de lesión, como síntomas y dilatación ventricular o disfunción sistólica. En caso de compromiso aórtico, sea dilatación, insuficiencia con disfunción de ventrículo izquierdo, disección o ruptura, se realiza reemplazo de válvula aórtica y aorta ascendente con tubo valvulado y reimplante de coronarias. Esta intervención conlleva riesgos de hemorragia, tromboembolismo y complicaciones del tratamiento anticoagulante ${ }^{31}$. En los últimos años se han introducido operaciones que reemplazan la raíz aórtica y aorta ascendente preservando la válvula cuando ésta aún no se ha deteriorado, siendo la más aceptada la técnica de Tirone David, que ha logrado buenos resultados con menor riesgo de hemorragia y tromboembolismo en el postoperatorio y evitando el uso de tratamiento anticoagulante. Sin embargo, se debe tener presente que por existir en pacientes con Marfán una alteración de la fibrilina, con el tiempo puede aparecer la lesión valvular, requiriéndose posteriormente el reemplazo de ésta ${ }^{5,30}$. La indicación de cirugía en dilatación aórtica se realiza en niños y adolescentes cuando el diámetro de la raíz aórtica es mayor al doble que lo normal para la edad y superficie corporal y en caso de progresión rápida (mayor de $1 \mathrm{~cm}$ por año) de la dilatación ${ }^{30,32}$. En adultos, se recomienda si es mayor a $55 \mathrm{~mm}$; si es mayor de $50 \mathrm{~mm}$ en pacientes con historia familiar de disección, con insuficiencia severa que requiere cirugía o con crecimiento rápido de raíz aórtica mayor de $2 \mathrm{~mm}$ por año; si es mayor a $45-50 \mathrm{~mm}$ cuando se ha planificado un reemplazo con preservación de válvula y con máximo de $44 \mathrm{~mm}$ en la mujer que desea un embarazo $0^{5,30,32,33}$. La dilatación aórtica es la indicación más común de cirugía en niños, seguida por la insuficiencia mitral. La disección es inusual y se requiere un cuidadoso seguimiento principalmente en los más jóvenes, ya que una segunda operación puede ser necesaria después de 10 años ${ }^{34}$. 
Factores pronósticos. La progresión del riesgo cardiovascular es mayor en niños con diagnóstico efectuado antes de los 10 años de edad y en los casos esporádicos ${ }^{14}$. En mujeres con Marfán esporádico, el compromiso cardiovascular es más precoz y con mayor riesgo de complicaciones y muerte al final de la segunda década ${ }^{35}$. Existe actualmente una mayor expectativa de vida por los beneficios de la cirugía, una mayor frecuencia de casos leves detectados por el uso precoz de medios diagnósticos y el uso de betabloqueadores $^{36}$. La insuficiencia aórtica aparece en general con dilatación mayor a $50 \mathrm{~mm}$ y el riesgo de disección con diámetro mayor a $60 \mathrm{~mm}^{8}$. Se ha estimado que la medición del radio aórtico (medición de dimensión máxima a nivel de senos de Valsalva en relación a edad y superficie corporal) mediante ecocardiografía, es un método adecuado para evaluar la existencia de dilatación aórtica y que valores mayores o iguales a 1,3 o incremento

\section{REFERENCIAS}

1. Lipscomb KJ, Clayton-Smith J, HaRris R. Evolving phenotype of Marfan's Syndrome. Arch Dis Child 1997; 76: 41-6.

2. RoBBins. Síndrome de Marfán. En: Patología Estructural y Funcional. 6르 Ed. McGraw-Hill. Interamericana. Madrid 2000. pág 159-160.

3. Jorde L, Carey J, Bamshad M, White R. Genética del Desarrollo. En: Genética Médica. 2ª Ed. Harcourt, Madrid, 2000. Pág. 204-220.

4. RoвBins. Elastina, fibrilina y fibras elásticas. En: Patología Estructural y Funcional. Cotran, Kumar y Collins. $6^{\underline{a}}$ Ed. Madrid, McGraw-Hill Interamericana, 2000. Pág. 106.

5. Mejjoom LJ, Nowen GJ, Mulder BJM. Prevention of cardiovascular complications in the Marfan Syndrome. Vascular Disease Prevention 2004; 1: 7986.

6. Gigante A, Chimemi C, Greco F. Changes of elastic fibers in musculoskeletal tissues of Marfan Syndrome: a possible mechanism of joint laxity and skeletal overgrowth. J Pediatr Orthop 1999; 19: 283-8. mayor a 5\% anual tienen más riesgo de complicaciones ${ }^{37,38}$.

Avances. Está en etapa de investigación la realización de estudios genéticos para el diagnóstico preimplantación y prenatal, que tendnían relevancia en países donde el aborto está legalizado y en casos de familias con mutación conocida ${ }^{5,39,40}$. El diagnóstico molecular postnatal es útil para identificar los individuos con riesgo de Marfán, principalmente en familias con manifestaciones variables, casos esporádicos por no tener el antecedente familiar y niños, ya que en ellos sus manifestaciones iniciales pueden ser incompletas. Un aporte para un mejor estudio cualitativo y cuantitativo de las lesiones cardiovasculares se ha obtenido a través de la ecocardiografía transesofágica (método de primera línea para enfermedades del arco aórtico), de la TAC (para obtener una visión tridimensional más precisa de las lesiones) y de la RM que permite mejor resolución de la anatomía y funcionalidad.

7. Robinson L Síndrome de Marfán. En: Nelson Tratado de Pediatría. Vol. No 2. 15a Ed. McGrawHill Interamericana, Madrid 1997, pág. 2468-69.

8. REED P. Genetics and Cardiovascular Disease. En: Braunwald ed. Heart Disease. WB Saunders $4^{\text {a }}$ Ed. Philadelphia, 1992, Vol. Nº 2; pág. 1622-49.

9. Ng DK, Chau KW, Black C, Thomas TM, MaK KL, BOXER M. Neonatal Marfan Syndrome: a case report. J Paediatr Child Health 1999; 35: 321-3.

10. Amaral FT, Carvalho SR, Granzotti Ja, Vieira LH, PinA Neto JM, Nunes MA. Neonatal heart failure and Marfan Syndrome. Arq Bras Cardiol 1996; 67: 355-7.

11. Luciani GB, Faggian G, Mazzucco A. Replacement of the descending thoracic aorta for massive aneurysm in neonatal Marfan Syndrome. J Card Surg 1994; 9: 109-14.

12. Buntinx IM, Wilems PJ, Spttaels SE, Van Reempst PJ, De Papepe AM, Dumon JE. Neonatal Marfan Syndrome with congenital arachnodactyly, flexion contractures, and severe cardiac valve insufficiency. J Med Genet 1991; 28: 267-73.

13. Geva T, Sanders S, Diogenes M, Rockenmacher $S$, Van PraAgh R. Two-dimensional and doppler 
echocardiographic and pathologic characteristics of the infantile Marfan Syndrome. Am J Cardiol 1990; 65: 1230-7.

14. Geva T, Hegesh J, Frand M. The clinical course and echocardiographic features of Marfan's Syndrome in childhood. AJDC 1987; 141: 1179-82.

15. De Paepe A, Devereux RB, Dietz HC, Hennekam R, Pyeritz R. Revised diagnostic criteria for the Marfan Syndrome. Am J Med Genetics 1996; 62: 417-26.

16. Yalcin F, Thomas JD, Homa D, Flachskampf FA. Transesophageal echocardiography: first-line imaging for aortic diseases. Cleve Clin J Med 2000; 67: 417-8, 421-8.

17. Ríos G. Resonancia Magnética. Métodos de exploración del Aparato Circulatorio. En: Rojas Villegas, Francisco. Enfermedades del corazón y de los vasos. 3a Ed. Edit Mediterráneo 2000. Santiago, Chile, pág, 246-63.

18. Fattori R, Bacchi Reggiani L, Pepe G, Napol G, Bna C, Celetti F et al. Magnetic resonance imaging evaluation of aortic elastic properties as early expression of Marfan Syndrome. J Cardiovasc Magn Reson 2000; 2: 251-6.

19. Mejboom LJ, Groenink M, Van Der Wall EE, Romkes H, StojeR J, Mulder BJ. Aortic root asymmetry in Marfan patients; evaluation by magnetic resonance imaging and comparison with standard echocardiography. Int J Card Imaging 2000; 16: 161-8.

20. Groenink GJ, Tujsen JG, Van Der Wall EE, Mulder BJ. Aortic stiffness and diameter predict progressive aortic dilatation in patients with Marfan Syndrome. Eur Heart J 2004; 25: 1146-52.

21. Robergen SA, De Roos A. Congenital heart disease. Evaluation of anatomy and function by MRI. Herz 2000; 25: 365-83.

22. Habermann CR, Weiss F, Schoder V, Cramer MC, Kemper J, WittKugel O et al. MR evaluation of dural ectasia in Marfan Syndrome: reassessment of the established criteria in children, adolescents, and young adults. Radiology 2005; 234: 535-41. Epub 2004 Dec 22.

23. Miledge JT, Ades LC, CoOper MG, Jaumees A, ONIKUL E. Severe spontaneus intracraneal hypotension and Marfan Syndrome in an adolescent. J Paediatr Child Health 2005; 41: 86-71.

24. Rosser T, Finkel J, Vezina G, Majd M. Postural headache in a child with Marfan Syndrome: case report and review of the literature. J Child Neurol 2005; 20: 153-5.
25. Rose PS, Levy HP, Ahn NU, Sponseler PD, Magyari T, Davis J ET AL. A comparison of the Berlin and Ghent nosologies and the influence of dural ectasia in the diagnosis of Marfan Syndrome. Genet Med 2000; 2: 278-82.

26. Committee on Genetics, American Academy of Pediatrics. Health Supervision for Children with Marfan Syndrome. Pediatrics 1996; 98: 978-82.

27. Maron B, Ackerman M, Nishimura R, Pyeritz R, Towbin J, Udelson J. Task Force 4: HCM and Others Cardiomyopathies, Mitral Valva Prolapse, Myocarditis, and Marfan Syndrome. JACC 2005; 45: 1340-5.

28. Shores J, Berger KR, Murphy EA, Pyeritz R. Progression of aortic dilatation and the benefit of long-term ß-adrenergic blockade in Marfan's Syndrome. N Engl J Med 1994; 330: 1335-41.

29. Groenink M, De Roos A, Muider BJ, Spaan J, Van DeR WaL E. Changes in aortic distensibility and pulse wave velocity assessed with magnetic resonance imaging following beta-blocker therapy in the Marfan Syndrome. Am J Cardiol 1988; 82: 203-8.

30. Milewicz D, Dietz H, Miuer C. Aortic disease in patients with Marfan Syndrome. Circulation 2005; 111: 150-7.

31. Lemaire SA, COSEW JS. Aortic root surgery in Marfan Syndrome: current practice and evolving techniques. J Card Surg 1997; 12 (2 Suppl): 13741.

32. CarRel T. Cardiovascular surgery in Marfan Syndrome: A review with case examples. Schweiz Med Wochenschr 1997; 127: 992-1006.

33. SteCKMEIER B. Epidemiology of aortic disease: aneurysm, dissection, occlusion. Radiology 2001; 41: 624-32.

34. Giwnov AM, Zehr KJ, Redmond JM, Gott V, Deitz H, ReITZ B ET AL. Cardiac operations in children with Marfan's Syndrome: indications and results. Ann Thorac Surg 1997; 64: 1140-5.

35. Van Karnebeek CD, NaefF MS, Mulder BJ, HenneKam M, OfFRinga M. Natural history of cardiovascular manifestations in Marfan Syndrome. Arch Dis Child 2001; 84: 129-37.

36. Silverman DI, Burton KJ, Gray J, Bosner M, Kouchoukos N, Roman M ET aL. Life expectancy in the Marfan Syndrome. Am J Cardiol 1995; 75: 157-60.

37. Legget ME, Unger TA, O'suLuvan CK, Zwink T, Bennett R, Byers P et Al. Aortic root complications 
in Marfan's Syndrome: identification of a lower risk group. Heart 1996; 75: 389-95.

38. Smith FC, Kavey RE. A new on-line method for predicting aortic root dilation durign two-dimensional achocardiography in pediatric patients with Marfan Syndrome using the sinus of valsalva to annulus ratio. Pediatr Cardiol 2003; 24: 118121. Epub 2002 Oct 10.
39. Loeys B, NuYtinck L, Van Acker P, Walraedt S, Bonduelie M, SERMon K ET AL. Strategies for prenatal and preimplantation genetic diagnosis in Marfan Syndrome (MFS). Prenat Diagn 2002; 22: 22-8.

40. Toudjarska I, Kilpatrick MW, Lembessis P, Carra S, HaRTon GL, Sisson ME ET AL. Novel approach to the molecular diagnosis of Marfan Syndrome: application to sporadic cases and in prenatal diagnosis. Am J Med Genet 2001; 99: 294-302.

Agradecimientos

Agradecemos la especial colaboración del Dr. Eduardo Turner, Cardiocirujano del Hospital del Tórax, Santiago y Dr. Felipe Heusser, Cardiólogo Infantil de la Pontificia Universidad Católica de Chile, por sus aportes en la revisión de este tema, y a la Sra. Cristina Miranda, Asistente de Servicios del Centro Saval Temuco, por su invaluable apoyo y asesoría. 